\title{
उMR
}

\section{Effect of luteolin on gene expression in mouse H22 hepatoma cells}

\author{
J.X. Niu' ${ }^{1}$, H.P. Guo ${ }^{2}$, H.M. Gan ${ }^{3}$, L.D. Bao ${ }^{4}$, J.J. Ren ${ }^{1}$ \\ ${ }^{1}$ Department of General Surgery, \\ Affiliated Hospital of Inner Mongolia Medical University, Hohhot, China \\ ${ }^{2}$ Department of Physical Examination, Binzhou People's Hospital, Shandong, \\ China \\ ${ }^{3}$ Intensive Care Unit, Binzhou People's Hospital, Shandong, China \\ ${ }^{4}$ Department of Pharmacy, \\ Affiliated Hospital of Inner Mongolia Medical University, Hohhot, China \\ Corresponding authors: J.J. Ren / L.D. Bao \\ E-mail: renjianjun237@163.com / Baolidao237@qq.com
}

Genet. Mol. Res. 14 (4): 14448-14456 (2015)

Received June 3, 2015

Accepted September 8, 2015

Published November 18, 2015

DOI http://dx.doi.org/10.4238/2015.November.18.7

\begin{abstract}
The purpose of our study was to observe the effects of luteolin on the expression of the genes ICAM-1, LFA-3, and PCNA in H22 hepatoma tissue. Sixty ICR (Institute of Cancer Research) mice with $\mathrm{H} 22$ hepatoma were randomly divided into five groups: a normal saline control group, low-, medium-, and high-dose luteolin groups, and a cyclophosphamide group. The mice were euthanized the day after administration withdrawal and subcutaneous tumor tissue was extracted. Quantitative fluorescence RT-PCR was used to detect the expression of ICAM-1, LFA-3, and PCNA in H22 hepatoma tissue in the mice. Luteolin was found to up-regulate the expression of ICAM-1 in H22 hepatoma tissue, of which the middle-dose group had the most obvious effect, showing a significant difference $(\mathrm{P}<0.01)$ as compared to the normal saline group. Each dose group of luteolin significantly
\end{abstract}


down-regulated the expression of $L F A-3$ in $\mathrm{H} 22$ hepatoma tissue, showing significant differences as compared to the saline control group $(\mathrm{P}<0.01)$. The medium- and high-dose luteolin groups significantly reduced the expression of PCNA in H22 hepatoma tissue of ICR mice, where the effect of the high-dose group was the most obvious, and the difference between the two luteolin groups and the normal saline group was statistically significant $(\mathrm{P}<0.01)$. Luteolin may inhibit tumor angiogenesis and tumor cell proliferation by down-regulation of $L F A$ 3 and PCNA and up-regulation of ICAM-1 in tumor tissue of tumorbearing mice, thereby achieving its anti-tumor effect.

Key words: Luteolin; H22 hepatoma; Tumor tissue; Gene expression

\section{INTRODUCTION}

Traditional Chinese medicine theory holds that the pathogenesis of primary liver cancer is the stagnation of qi, blood, damp, heat, stasis, and toxins, which mostly contribute to vital qi deficiency, yin-yang disharmony, disorder of qi and blood, liver qi stagnation, spleen insufficiency, damp retention, phlegm coagulation, and blood stasis; and exogenous pathogenic factors of six climatic conditions in excess for transformation into heat in the course of time and toxic heat accumulation; internal damage by excess of seven emotions and emotional depression (Li et al., 2012; Liao et al., 2012). Luteolin can reduce heat and toxicity, remove jaundice, and eliminate edema, nourishing the liver and relieving pain (Liu et al., 2014). The traditional medicines Rhizoma sparganii, Curcuma zedoary, and Curcuma wenyujin typically enter the liver meridian and can promote the circulation of qi, disrupt blood stasis, remove qi stagnation, and prevent pain (He et al., 2014).

Liver cancer is usually accompanied by the symptoms of spleen-stomach deficiency and indigestion due to retention of food, so the traditional medicines mentioned above are supplemented with dried tangerine peel, Pericarpium citri Reticulatae viride, Coke hawthorn, and baked endothelium corneum gigeriae galli. These additional treatments help to regulate qi-flowing in order to strengthen the spleen, relieve flatulence, and aid transportation and transformation, which can significantly improve clinical symptoms, enhance patients' quality of life, and prolong survival time (Hsu et al., 2008; Liu et al., 2011). All these medicines enter the spleen meridian. The clinical efficacy of luteolin has been unanimously endorsed by doctors and patients since its clinical application.

In recent years, with the development of molecular biology, cell biology, and genetics, research on the mechanisms of traditional Chinese medicine in the treatment of liver cancer has advanced to the level of gene and cell signal transduction (Li and Ling, 2012; Zhang et al., 2012). A growing number of molecular anti-tumor mechanisms of traditional Chinese medicine have revealed that these medicines may be organically combined according to the targets of action based on syndrome differentiation for treatment using traditional Chinese medicine to improve the therapeutic effects of these medicines on the treatment of liver cancer. Studies have shown that luteolin has a significant tumor-inhibitory effect in mice with H22 hepatoma and S180 sarcoma; however, the exact mechanism is not yet clear (Ouyang et al., 2011). In this study, quantitative PCR was used to evaluate the effect of luteolin on the expression of the ICAM-1, LFA-3, and PCNA in H22 hepatoma tissue of mice and to further explore its molecular mechanism in 
order to provide experimental evidence for the clinical application of this medicine.

\section{MATERIAL AND METHODS}

\section{Animals and cell line}

The H22 hepatoma cell line was purchased from Bio-Rad Life Sciences Development Co., Ltd. (Beijing, China). Specific-pathogem-free Institute of Cancer Research (SPF ICR) mice aged 5 weeks old and weighing $20 \pm 1 \mathrm{~g}$ were purchased from the Laboratory Animal Science Department of Capital Medical University (Beijing, China), with the certificate No. of conformity of SCXK (Beijing) 2012-0058.

\section{Drugs}

Luteolin (ComWin Biotech Co., Ltd., Beijing, China) was prepared as a solution at a concentration of $0.5 \mathrm{~g} / \mathrm{mL}$, and then appropriate concentrations were prepared according to the dosage and volume of administration. Cyclophosphamide (CTX) for injection was purchased from Hengrui Medicine Co., Ltd. (Jiangsu, China) lot No. 20120823.

\section{Reagents}

TRIzol reagent was purchased from Santa Cruz (CA, USA), and mice ICAM-1, LFA3, and PCNA fluorescence quantitative RT-PCR detection kits were purchased from Ruixianghe Biotech Co., Ltd. (Beijing, China)

\section{Model establishment, grouping, and administration methods}

Sixty ICR male mice, each with a body weight of $20 \pm 1 \mathrm{~g}$, were selected. The ascitic fluid of mice that were to be inoculated with the H22 tumor strain under sterile conditions for 5 days was sampled (the ascitic tumor fluid was thick and ivory in color, yellow or red ascitic fluid was discarded) and diluted with normal saline to adjust the cell concentration to $1 \times 10^{5}$ $\mathrm{mL}$. The mice were subcutaneously inoculated in the right axilla of the forelimb with $0.5 \mathrm{~mL}$ ascitic tumor fluid for each. After inoculation, the mice were randomly divided into five treatment groups: the normal saline control group, CTX chemotherapy group, low-dose luteolin group, middle-dose luteolin group, and high-dose luteolin group ( $\mathrm{N}=12$ per group). After 24 $\mathrm{h}$ of inoculation, the negative control group was intragastrically administrated with $10 \mathrm{~mL} /$ $\mathrm{kg}$ saline once a day; the positive control group was intraperitoneally injected with $10 \mathrm{mg} / \mathrm{kg}$ CTX once a day; and the low-, middle-, and high-dose luteolin groups were intragastrically given 10,20, and $30 \mathrm{~g} / \mathrm{kg}$ luteolin solution, respectively, once a day. The administration was withdrawn after 14 days. The mice were euthanized the day after withdrawal and the subcutaneous tumor body was extracted, quickly stored in liquid nitrogen, and then transferred to a low-temperature refrigerator at $-35^{\circ} \mathrm{C}$ until required.

\section{Quantitative fluorescence RT-PCR}

Total mRNA extraction from tumor tissue was undertaken as follows: 1) $100 \mathrm{mg}$ stored tumor tissue was sampled and placed in a 2-mL centrifuge tube with $1 \mathrm{~mL}$ TRIzol 
for full homogenation, and then left statically at $24^{\circ} \mathrm{C}$ for $10 \mathrm{~min}$; 2) $0.5 \mathrm{~mL}$ chloroform was added, the sample was oscillated for $30 \mathrm{~s}$, and then left to stand statically for $3 \mathrm{~min} ; 3$ ) the sample was centrifuged at $4^{\circ} \mathrm{C}$ and $3000 \mathrm{rpm}$ for $10 \mathrm{~min}$ and the supernatant was extracted; 4) $0.5 \mathrm{~mL}$ isopropanol was added and the liquid was mixed in the tube gently and left to stand statically at room temperature for $5 \mathrm{~min} ; 5$ ) the sample was centrifuged at $4^{\circ} \mathrm{C}$ and $3000 \mathrm{rpm}$ for $10 \mathrm{~min}$ and then the supernatant was discarded; 6) $1 \mathrm{~mL} 75 \%$ ethanol was added to gently wash the precipitation and the sample was centrifuged at $4{ }^{\circ} \mathrm{C}$ and $3000 \mathrm{rpm}$ for $10 \mathrm{~min}$, and then the supernatant was discarded; 7) the sample was air dried and an appropriate amount of DEPC $\mathrm{H}_{2} \mathrm{O}$ was added for dissolution (solubilize at $60^{\circ} \mathrm{C}$ for $5 \mathrm{~min}$ ). RNA content and purity (OD value at $260 \mathrm{~nm}$ ) were measured using a UV spectrophotometer and the samples were stored at $-35^{\circ} \mathrm{C}$ until required.

Reverse transcription was performed according to the manufacturer protocol using the following reaction conditions: $37^{\circ} \mathrm{C}$ for $30 \mathrm{~min}$ and $95^{\circ} \mathrm{C}$ for $2 \mathrm{~min}$. Real-time PCR amplification was conducted according to the manufacturer protocol using the following reaction conditions: $93^{\circ} \mathrm{C}$ denaturation for $2 \mathrm{~min}$, then $93^{\circ} \mathrm{C}$ for $30 \mathrm{~s}$ and $55^{\circ} \mathrm{C}$ for $45 \mathrm{~s}$ for 30 cycles in total.

The PCR primer sequences are as follows: ICAM-1: forward primer: 5'-AGCGGGCC CTTACGACATA-3'; reverse primer: 5'-TCACATACGCTCGATACCGTC-3'; probe primer: 5'-AGCCACCTCGTGAGTGCCGGCAGA-3'. LFA-3: forward primer: 5'-GGCAGTCCAC ACGAAGCT-3'; reverse primer: 5'-CAGGCAGTAGGCAGGGAC-3'; probe primer: 5'-AGT ACCACGGAGGTGCGCGCTC-3'. PCNA: forward primer: 5'-ACGCGACTACCGTATAT A-3'; reverse primer: 5'-GACAGACTTGGGACGGCAAG-3'; probe primer: 5'-TCACCCGG ACAGCTGGGAATCGAGA-3' (Hou et al., 2010).

Positive standard points were selected for analysis. A correlation coefficient $\left(\mathrm{R}^{2}\right)$ greater than 0.95 indicated a good linear relationship, which could then be used as a quantitative standard curve. The absolute quantitative values for the quantitative fluorescence reaction of samples could then be obtained according to the standard curve (copy number of cDNA concentration $/ \mu \mathrm{L})$.

\section{Statistical analysis}

All data were analyzed using SPSS 14.0. Measurement data are reported as means \pm standard deviation, and the differences between groups were analyzed using $t$-tests.

\section{RESULTS}

\section{Effects of luteolin on ICAM-1 mRNA expression in hepatoma tissue}

The standard curve of the quantitative fluorescence RT-PCR was:

$$
Y=-3.5183 \operatorname{LgX}+39.6239
$$

(Equation 1)

$\mathrm{R}^{2}=0.9964$, which complies with the correlation coefficient of $\mathrm{R}^{2}>0.95$ in the manufacturer protocol, showing a good linear relationship (Figure 1). 


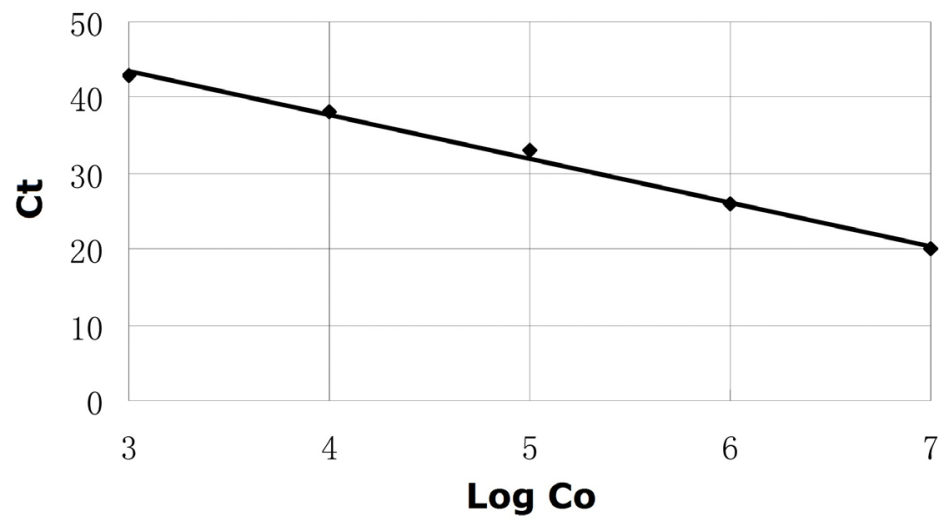

Figure 1. Standard curve of ICAM-1 expression in hepatoma tissue from mice.

ICAM- 1 expression was the highest in tissue from the middle-dose group, followed by the high-dose group, and the CTX chemotherapy group, successively. Expression of ICAM-1 in the low-dose group and the normal saline control group was similar and the lowest of all groups. ICAM- 1 expression was significantly higher in the middle-dose group, the high-dose group, and the CTX chemotherapy group compared to the normal saline control group $(\mathrm{P}<0.01$; Table 1$)$.

$\begin{aligned} & \text { Table 1. Quantitative fluorescence RT-PCR results of ICAM-1 expression in hepatoma tissue from mice } \\
& \text { subjected to five treatments. }\end{aligned}$
\begin{tabular}{lcc}
\hline Treatment group & Dose of saline, CTX, or luteolin & cDNA concentration (copy number $/ \mu \mathrm{L}$ ) \\
\hline Normal saline control & $10 \mathrm{~mL} / \mathrm{kg}$ & $1362.6 \pm 267.5$ \\
Cyclophosphamide (CTX) chemotherapy & $10 \mathrm{mg} / \mathrm{kg}$ & $8539.4 \pm 953.8^{*}$ \\
Low-dose luteolin & $10 \mathrm{~g} / \mathrm{kg}$ & $1434.8 \pm 284.1$ \\
Medium-dose luteolin & $20 \mathrm{~g} / \mathrm{kg}$ & $18649.5 \pm 1652.6^{*}$ \\
High-dose luteolin & $30 \mathrm{~g} / \mathrm{kg}$ & $9928.4 \pm 1123.7^{*}$ \\
\hline
\end{tabular}

Data are reported as means $\pm \mathrm{SD}$. *Significant difference compared to the normal saline control group, $\mathrm{P}<0.01$.

\section{Effects of luteolin on $L F A-3$ mRNA expression in hepatoma tissue}

The standard curve of the quantitative fluorescence RT-PCR was:

$$
\mathrm{Y}=-3.2675 \operatorname{LgX}+38.6492
$$

$\mathrm{R}^{2}=0.9958$, which complies with the correlation coefficient $\mathrm{R}^{2}>0.95$ in the manufacturer protocol, showing a good linear relationship (Figure 2).

The descending order of $L F A-3$ expression in the treatment groups was as follows: normal saline control group $>$ low-dose group $>$ middle-dose group $>$ CTX treatment group $>$ highdose group. A significant difference in expression was observed between the low-dose group, the middle-dose group, the high-dose group, and the CTX chemotherapy group compared with the normal saline control group $(\mathrm{P}<0.01$; Table 2$)$. A significant difference in expression was also observed between the high-dose group and the CTX chemotherapy group $(\mathrm{P}<0.01$; Table 2$)$. 


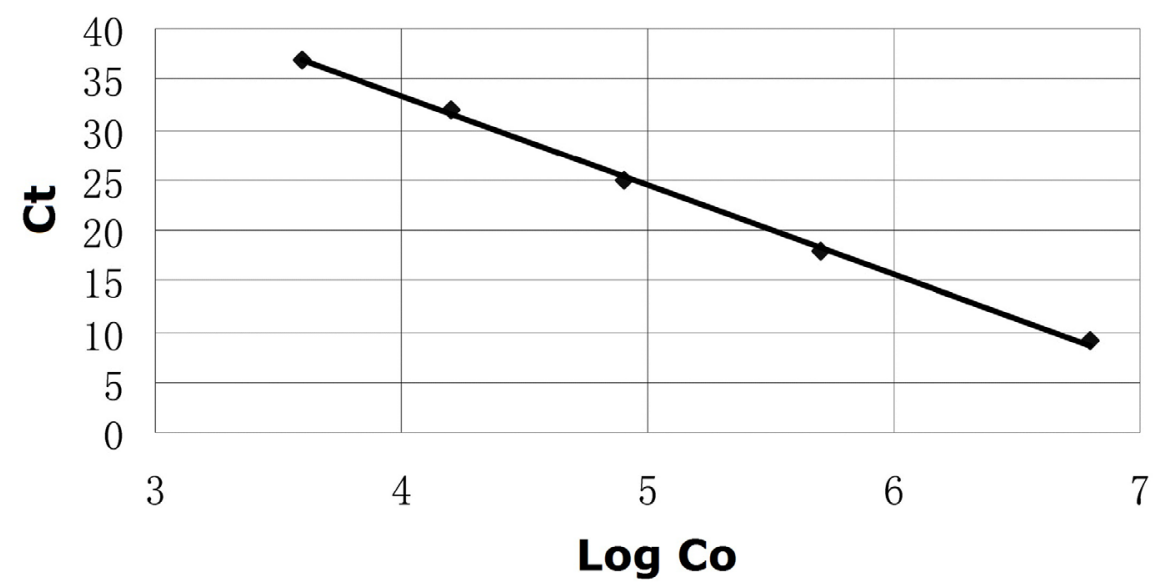

Figure 2. Standard curve of $L F A-3$ expression in hepatoma tissue from mice.

Table 2. Quantitative fluorescence RT-PCR results of $L F A-3$ expression in hepatoma tissue from mice subjected to five treatments.

\begin{tabular}{lcc}
\hline Treatment group & Dose of saline, CTX, or luteolin & cDNA concentration (copy number/ $\mu \mathrm{L})$ \\
\hline Normal saline control & $10 \mathrm{~mL} / \mathrm{kg}$ & $183545.2 \pm 14125.2$ \\
Cyclophosphamide (CTX) chemotherapy & $10 \mathrm{mg} / \mathrm{kg}$ & $15362.9 \pm 1683.5^{*}$ \\
Low-dose luteolin & $10 \mathrm{~g} / \mathrm{kg}$ & $95365.7 \pm 6251.8^{*}$ \\
Medium-dose luteolin & $20 \mathrm{~g} / \mathrm{kg}$ & $35418.9 \pm 2646.1^{*}$ \\
High-dose luteolin & $30 \mathrm{mg} / \mathrm{kg}$ & $2641.6 \pm 396.8^{*}$ \\
\hline
\end{tabular}

Data are reported as means \pm SD. *Significant difference compared to the normal saline control group, $\mathrm{P}<0.01$.

\section{Effects of luteolin on PCNA mRNA expression in hepatoma tissues}

The standard curve of the quantitative fluorescence RT-PCR was:

$$
\mathrm{Y}=-3.1686 \mathrm{LgX}+37.1823
$$

(Equation 3)

$\mathrm{R}^{2}=0.9936$, which complies with the correlation coefficient $\mathrm{R}^{2}>0.95$ in the manufacturer protocol, showing a good linear relationship (Figure 3).

$P C N A$ expression was the highest in the normal saline control group, followed by the low-dose and middle-dose groups, and expression in the high-dose group and the CTX chemotherapy group was similar and the lowest of all groups. A significant difference in expression of PCNA was observed between the high-dose group and the CTX chemotherapy group compared with the normal saline control group $(\mathrm{P}<0.01$; Table 3$)$, while there was no significant difference in expression between the high-dose group and the CTX chemotherapy group $(\mathrm{P}>0.05$; Table 3$)$. 


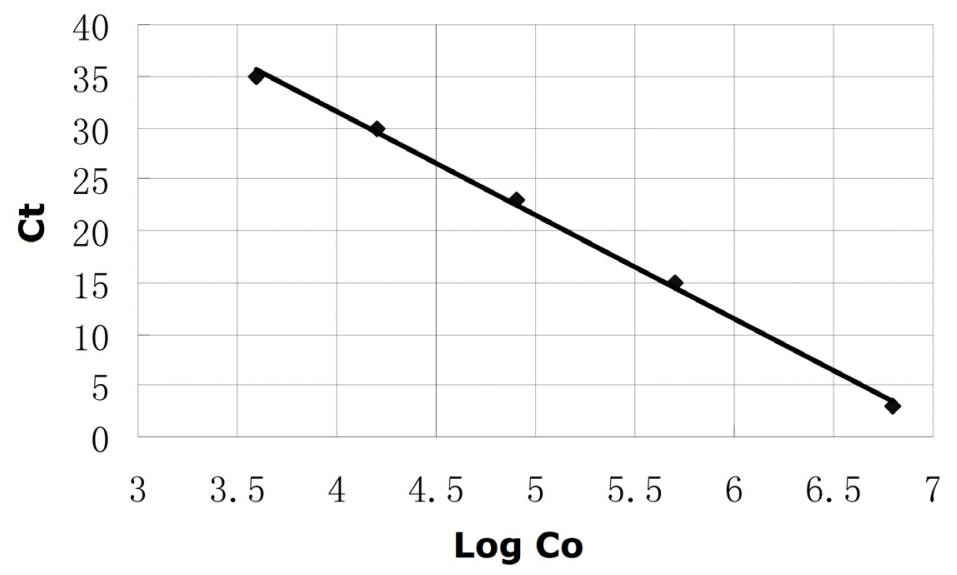

Figure 3. Standard curve of $P C N A$ expression in hepatoma tissue from mice.

Table 3. Quantitative fluorescence RT-PCR results of PCNA expression in hepatoma tissue from mice subjected to five treatments.

\begin{tabular}{lcc}
\hline Treatment group & Dose of saline, CTX, or luteolin & cDNA concentration (copy number/ $\mu \mathrm{L}$ ) \\
\hline Normal saline control & $10 \mathrm{~mL} / \mathrm{kg}$ & $15853.9 \pm 2511.6$ \\
Cyclophosphamide (CTX) chemotherapy & $10 \mathrm{gg} / \mathrm{kg}$ & $13364.8 \pm 1738.4$ \\
Low-dose luteolin & $10 \mathrm{~g} / \mathrm{kg}$ & $9824.5 \pm 1251.4^{*}$ \\
Medium-dose luteolin & $20 \mathrm{~g} / \mathrm{kg}$ & $3962.8 \pm 625.5^{*}$ \\
High-dose luteolin & $30 \mathrm{~g} / \mathrm{kg}$ & $3748.1 \pm 568.8^{*}$
\end{tabular}

Data are reported as means $\pm \mathrm{SD}$. * Significant difference compared to the normal saline control group, $\mathrm{P}<0.01$.

\section{DISCUSSION}

With the use of genomic and proteomic technologies in the early detection, postoperative recurrence, metastasis monitoring, positive high sensitivity, and specific diagnosis of liver cancer, it has been found that the progress of liver cancer is closely correlated with the over-expression of a variety of growth factors, such as ICAM-1, LFA-3, and PCNA in liver tissue, which has complex molecular mechanisms (Kawai et al., 2009; Ueda et al., 2009). Experimental studies have shown that luteolin has a significant tumor-inhibitory effect in mice with H22 hepatoma and S180 sarcoma. Low-, medium- and high-dose luteolin groups can all prolong the survival days of mice with $\mathrm{H} 22$ hepatoma ascetic tumor and can down-regulate the expression of CyclinD1 mRNA in tumor tissue (Pan et al., 2012). The current study further examined the effect of luteolin on the expression of the ICAM-1, LFA-3, and PCNA in tumor tissue of mice with $\mathrm{H} 22$ hepatoma.

ICAM is a multifunctional cytokine, involved in the process of cell cycle regulation, wound healing, angiogenesis, cell growth, differentiation, apoptosis, etc. (Tse et al., 2009). ICAM-1 can play a tumor suppressor role by inhibiting cell growth and promoting differentiation, and activated ICAM-1 functions through the mediation of its corresponding receptor (Turner et al., 2011). Recent studies suggest that decreased expression of the transforming growth factor ICAM-1 is an important alteration in the negative growth regulation of tumor 
cells escaping from immune surveillance, which may lead to uncontrolled proliferation and canceration (Zapolska-Downar and Naruszewicz, 2009). Studies have found that, compared to hepatitis or liver fibrosis patients, expression of $I C A M-1$ in patients with liver cancer is significantly reduced, and $I C A M-1$ expression in hepatoma tissue is also significantly lower than that of normal tissue, while ICAM- 1 expression is higher in hepatoma tissue with high differentiation, small tumor size, low metastasis, and long recurrence interval $(\mathrm{P}<0.05)$ (Rizk and Derbala, 2013). This indicates that $I C A M-1$ expression is reduced in hepatoma tissue, leading to a negative regulatory barrier of liver cancer cells, which may be one of the key factors for hepatocellular canceration. ICAM-1 expression, which is negatively correlated with the malignant progression of liver cancer, has potential significance in the treatment of this disease (Tu et al., 2011).

The nuclear transcription factor LFA-3 can participate in many stages in the occurrence and development of liver cancer, such as blocking gene expression related to apoptosis, promoting cell proliferation, mediating tumor development, angiogenesis and metastasis, promoting tumor cell invasion and spreading, as well as the generation of tumor drug-resistance, and it is also a major regulatory factor of LFA synthesis (Metzler et al., 2008). Studies have found that LFA-3 exhibited significantly higher expression in hepatoma tissue, suggesting that $L F A-3$ may play the role of oncogene in the occurrence and development of liver cancer (Franciszkiewicz et al., 2013).

PCNA, as a major pro-angiogenic factor in tumor tissue, can directly stimulate the proliferation of endothelial cells through specific receptors, stimulating production of plasminogen activator and collagenase, and increasing vascular permeability and promoting plasma fibrin exosmosis (Bologna-Molina et al., 2013). This not only promotes endothelial cell migration, which is conducive to angiogenesis, but also helps tumor cells shed into blood vessels or diffuse to matrices near fibrous and connective tissues, so as to create the conditions for tumor invasion and metastasis. Liver cancer is a malignancy with rich blood supply and high metastasis, and PCNA is closely related with tumor angiogenesis, proliferation, invasion, and metastasis (Margotta, 2012). Studies have shown that the expression rates of PCNA in liver cancer, nonencapsulated liver cancer, and liver cancer with extrahepatic metastasis were 63.9, 78.3, and 90.9\%, respectively. Under high expression of $P C N A$, hepatoma tissue can produce a large number of tumor blood vessels; at the same time, PCNA can also increase capillary permeability, thereby enabling tumor blood perfusion to be increased (Lee and Lim, 2009). The results of the current study show that each luteolin dose group up-regulates $I C A M-1$ expression and down-regulates the expression of $L F A-3$ and $P C N A$. This suggests that the tumor-inhibitory effect of luteolin is multi-channeled and multi-targeted, which not only affects the cycle-regulatory factors of tumor cells, but also inhibits tumor angiogenesis and tumor cell proliferation by regulating relevant vascular factors, ultimately achieving its anti-tumor effect. The in-depth mechanism of the tumor-inhibitory effect of luteolin needs to be further studied, while the results of this investigation provide experimental basis for the clinical application of this medicine.

\section{Conflicts of interest}

The authors declare no conflict of interest.

\section{ACKNOWLEDGMENTS}

Research supported by the One Million Technology Project of Inner Mongolia Medical University (\#NY2011bw004), the Medical and Health Research Project of Health Bureau 
of Inner Mongolia (\#2010038), the Doctoral Station Initiation Project of Inner Mongolia Medical University (\#Nyfybz2010003), and the Scientific Research Project of the Higher Education Institutions of Inner Mongolia Autonomous Region (\#NJZY13416).

\section{REFERENCES}

Bologna-Molina R, Mosqueda-Taylor A, Molina-Frechero N, Mori-Estevez AD, et al. (2013). Comparison of the value of PCNA and Ki-67 as markers of cell proliferation in ameloblastic tumors. Med. Oral Patol. Oral Cir. Bucal. 18: 174-179.

Franciszkiewicz K, Le Floc'h A, Boutet M, Vergnon I, et al. (2013). CD103 or LFA-1 engagement at the immune synapse between cytotoxic $\mathrm{T}$ cells and tumor cells promotes maturation and regulates T-cell effector functions. Cancer Res. 73: 617-628.

He XX, Wu QN, Wang XS, Chen GY, et al. (2014). Influence of different drying methods on the quality of Sparganii rhizoma based on multi-index evaluation. Zhong Yao Cai 37: 29-34.

Hou Y, Zhang H, Miranda L and Lin S (2010). Serious overestimation in quantitative PCR by circular (supercoiled) plasmid standard: microalgal pcna as the model gene. PLoS One 5: e9545.

Hsu YL, Kuo PL, Tzeng TF, Sung SC, et al. (2008). Huang-lian-jie-du-tang, a traditional Chinese medicine prescription, induces cell-cycle arrest and apoptosis in human liver cancer cells in vitro and in vivo. J. Gastroenterol. Hepatol. 23: e290-e299.

Kawai Y, Kaidoh M, Yokoyama Y, Sano K, et al. (2009). Chemokine CCL2 facilitates ICAM-1-mediated interactions of cancer cells and lymphatic endothelial cells in sentinel lymph nodes. Cancer Sci. 100: 419-428.

Lee SJ and Lim KT (2009). Phytoglycoprotein (24 kDa) inhibits expression of PCNA via PKCalpha and MAPKs in oxygen radical-stimulated Chang liver cells. J. Nutr. Biochem. 20: 96-105.

Li M, Qiao C, Qin L, Zhang J, et al. (2012). Application of traditional Chinese medicine injection in treatment of primary liver cancer: a review. J. Tradit. Chin. Med. 32: 299-307.

Li XQ and Ling CQ (2012). Chinese herbal medicine for side effects of transarterial chemoembolization in liver cancer patients: a systematic review and meta-analysis. Zhong Xi Yi Jie He Xue Bao 10: 1341-1362.

Liao YH, Lin CC, Li TC and Lin JG (2012). Utilization pattern of traditional Chinese medicine for liver cancer patients in Taiwan. BMC Complement. Altern. Med. 12: 146.

Liu G, Zhang Y, Liu C, Xu D, et al. (2014). Luteolin alleviates alcoholic liver disease induced by chronic and binge ethanol feeding in mice. J. Nutr. 144: 1009-1015.

Liu ML, Chien LY, Tai CJ, Lin KC, et al. (2011). Effectiveness of traditional Chinese medicine for liver protection and chemotherapy completion among cancer patients. Evid. Based Complement. Altern. Med. 2011: 291843.

Margotta V (2012). Relationships between seasonal thermal variations and cell proliferation in heterothermic vertebrates, as revealed by PCNA expression in the brain of adult Rana bergeri (Gunther, 1986). Ital. J. Anat. Embryol 117: 45-53.

Metzler B, Gfeller P, Wieczorek G and Katopodis A (2008). Differential promotion of hematopoietic chimerism and inhibition of alloreactive $\mathrm{T}$ cell proliferation by combinations of anti-CD40Ligand, anti-LFA-1, everolimus, and deoxyspergualin. Transpl. Immunol. 20: 106-112.

Ouyang H, Wang P, Meng Z, Chen Z, et al. (2011). Multimodality treatment of pancreatic cancer with liver metastases using chemotherapy, radiation therapy, and/or Chinese herbal medicine. Pancreas 40: 120-125.

Pan ZQ, Fang ZQ and Lu WL (2012). Analysis of the dynamic changes of blood hormone levels in H22 liver cancer mice of poisonous pathogenic factors syndromes to different degrees. Zhongguo Zhong Xi Yi Jie He Za Zhi 32: 1361-1365.

Rizk NM and Derbala MF (2013). Genetic polymorphisms of ICAM 1 and IL28 as predictors of liver fibrosis severity and viral clearance in hepatitis C genotype 4. Clin. Res. Hepatol. Gastroenterol. 37: 262-268.

Tse MC, Lane C, Mott K, Onlamoon N, et al. (2009). ICAM-5 modulates cytokine/chemokine production in the CNS during the course of herpes simplex virus type 1 infection. J. Neuroimmunol. 213: 12-19.

Tu CT, Han B, Liu HC and Zhang SC (2011). Curcumin protects mice against concanavalin A-induced hepatitis by inhibiting intrahepatic intercellular adhesion molecule-1 (ICAM-1) and CXCL10 expression. Mol. Cell. Biochem. 358: 53-60.

Turner NA, Das A, O'Regan DJ, Ball SG, et al. (2011). Human cardiac fibroblasts express ICAM-1, E-selectin and CXC chemokines in response to proinflammatory cytokine stimulation. Int. J. Biochem. Cell Biol. 43: 1450-1458.

Ueda R, Kohanbash G, Sasaki K, Fujita M, et al. (2009). Dicer-regulated microRNAs 222 and 339 promote resistance of cancer cells to cytotoxic T-lymphocytes by down-regulation of ICAM-1. Proc. Natl. Acad. Sci. U.S.A. 106: 10746-10751.

Zapolska-Downar D and Naruszewicz M (2009). Propionate reduces the cytokine-induced VCAM-1 and ICAM-1 expression by inhibiting nuclear factor-kappa B (NF-kappaB) activation. J. Physiol. Pharmacol. 60: 123-131.

Zhang YH, Qin X and Xu J (2012). Analysis of Chinese medical syndrome features of patients with primary liver cancer before and after transcatheter arterial chemoembolization. Zhongguo Zhong Xi Yi Jie He Za Zhi 32: 1171-1174. 